\title{
Metastasis of the gallbladder in clear cell renal carcinoma
}

\author{
ANDREA BOSCÀ ROBLEDO ${ }^{1,2}$, SEBASTIÁN BARBER MILLET ${ }^{1}$, JUAN F. ORBIS CASTELLANOS ${ }^{1}$, \\ EVA M. MONTALVÁ ORÓN ${ }^{1}$, JOSE V. SALOM FUSTER ${ }^{1}$ and MANUEL DE JUAN BURGUEÑO ${ }^{1}$ \\ ${ }^{1}$ Department of General Surgery, La Fe University Hospital, Valencia 46026, Spain \\ Received November 14, 2011; Accepted January 17, 2012
}

DOI: 10.3892/ol.2012.616

\begin{abstract}
Metastasis of the gallbladder due to renal cell tumors is rare. We present a case of gallbladder and metachronous left adrenal metastasis at six months follow-up, which demonstrates the importance of radiological tests and histology when making a definitive diagnosis. Clinical findings are not specific enough to arrive at a final diagnosis. However, immunohistochemistry is necessary to differentiate between primary and secondary metastatic tumors. Cholecystectomy should be performed to obtain a definitive diagnosis and to improve survival in cases of single lesions in the gallbladder.
\end{abstract}

\section{Introduction}

Clear cell renal carcinoma (RCC) is a rare tumor accounting for $3 \%$ of all malignancies in adults and $85 \%$ of primary renal tumors (1). Currently, its incidence is on the increase due to the increased number of incidental findings in imaging tests (2). RCC frequently metastasizes in the lungs, bones, lymph nodes and liver. Gallbladder metastasis is extremely rare, being found in approximately $0.6 \%$ of cases at autopsy (3). We report a case of gallbladder and metachronous contralateral adrenal metastasis. The study was approved by the ethics committee of La Fe University Hospital, and consent was obtained from the patient.

\section{Case report}

A 75-year-old female was incidentally diagnosed with right RCC following radiological tests requested for benign anorectal disease in March 2010. Computed tomography (CT) revealed a right renal mass of $11.3 \times 8.6 \times 12.2 \mathrm{~cm}$ with

Correspondence to: Ms. Andrea Boscà Robledo, Department of General Surgery, La Fe University Hospital, Bulevar sud, s/n, Valencia 46026, Spain

E-mail: andreabosca@comv.es

Present address: ${ }^{2} \mathrm{C} /$ Villa de Muro, $\mathrm{n}^{\circ} 2$, puerta 8, Valencia 46020, Spain

Key words: gallbladder metastasis, clear cell renal carcinoma, cholecystectomy areas of necrosis, calcification, increased neovascularization and exophytic growth towards the liver. In addition, lymph nodes were detected in the renal hilum and retroperitoneum, with uncertain infiltration of the renal vein and inferior vena cava without distant metastases. The tumor markers CEA and CA19.9 were within the normal range. A radical right nephrectomy was carried out through a right subcostal incision without demonstrable vascular invasion or metastases intraoperatively. Macroscopically, the renal lesion was yellowish in appearance, with undefined limits and a confluent nodular pattern with fibrous areas.

The patient's postoperative course was uneventful and systemic adjuvant therapy was not administered. The pathological study revealed a typical RCC with a solid growth pattern without invasion of the renal pelvic wall and tumor thrombosis in the renal vein. Surgical margins were tumor-free and all lymph nodes dissected were free of tumor infiltration, with stage pT3N0M0.

A CT scan was performed at 6 months which showed a nodular lesion of $3 \mathrm{~cm}$ in the left adrenal gland and a lobulated lesion in the gallbladder wall, with the suspected diagnosis of metastasis. Ultrasonography (US) revealed a heterogeneous solid nodular structure of $2 \mathrm{~cm}$ with poor delineation of the gallbladder wall. It was not possible to exclude infiltration of the liver parenchyma (Fig. 1). The extension study was completed with a thoracic CT, which revealed no pathological findings. Tumor markers were also within normal ranges.

A midline laparotomy was carried out and the patient underwent left adrenalectomy and cholecystectomy, with no complications during the postoperative period (Fig. 2). There was a polypoid lesion of the gallbladder without infiltration of the wall and the left adrenal nodule had a yellowish appearance with areas of cystic hemorrhage (Fig. 3A). Histological examination of the two specimens showed metastases of RCC.

At present, the patient is receiving corticosteroid replacement therapy with no adjuvant treatment. A CT scan four months after the surgery showed no signs of recurrence.

\section{Discussion}

Metastatic gallbladder lesions are infrequent; melanoma, stomach, pancreas, ovary, small bowel, biliary duct and breast carcinomas are those that have the highest probability of metastasis (4). Half of the cases are synchronic. However, RCC is the primary renal tumor that metastasizes most frequently in the gallbladder (5). 


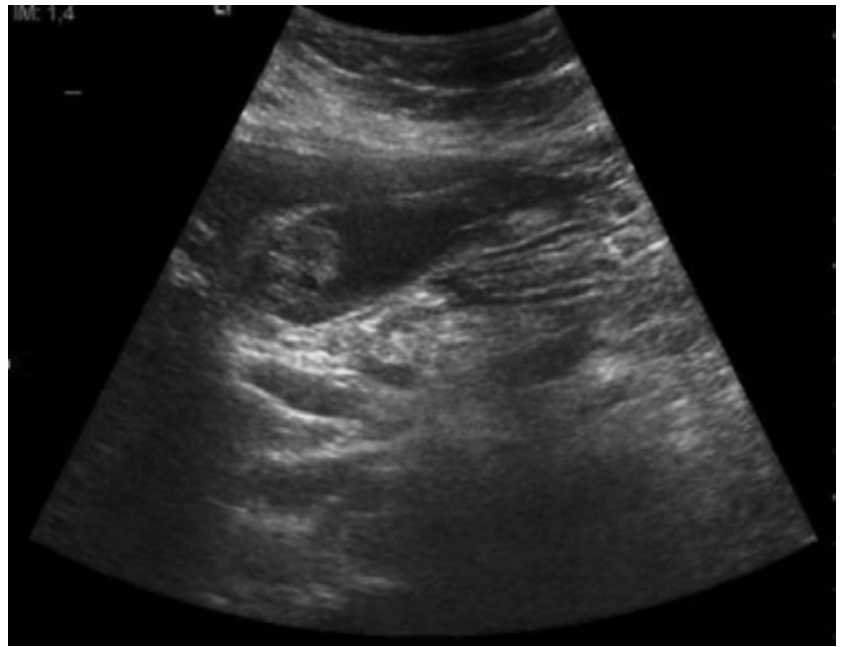

Figure 1. Ultrasonography image. Heterogeneous nodule attached to the gallbladder wall without posterior echogenic shadowing, with well-defined edges and adjacent wall thickening.

Clinical findings are not specific enough to arrive at a final diagnosis. Primary tumors prevail in women over 65 years of age with biliary lithiasis (6). Metastatic tumors usually appear in 39 to 84-year-old males, who have a personal history of cancer without previous biliary pathology (4).

Imaging diagnosis may not be conclusive in making a differential diagnosis between primary and secondary tumors. US is the initial approach in the diagnosis of gallbladder tumors, and this may be further improved with contrast US. Metastases can appear under different hyperechoic masses bigger than $1 \mathrm{~cm}$ in diameter, close to the gallbladder wall without posterior acoustic shadowing (7). In primary tumors, a solid mass is observed, occupying the entire wall thickness, or a polypoid lesion with increased vascularization (8). A radiological finding that can help to differentiate between a primary gallbladder tumor and metastasis in the CT scan is the invasion of the mucosal layer. If the mucosa is not infiltrated, indicating an invasion from the serosa layer, the primary gallbladder tumor can be excluded. In tumoral gallbladder invasion by contiguous tumors, a great mass without clear limits is observed (7).

There is no evidence to suggest any benefit from using positron emission tomography-computed tomography (PET-CT) to detect gallbladder metastases, which may be due to the low number of cases reported (9). The utility of this test for future diagnoses and follow-up should be validated.

The specific diagnosis of polypoid gallbladder masses is problematic. It is necessary to make differential diagnosis between cholesterol polyps, gallbladder clear cell tumor, carcinoid and paragangliomes (4). Immunohistochemistry is also necessary to differentiate between primary and secondary metastatic tumors. In primary tumors, extremely high CEA and CK7 levels, and moderately raised levels of CK10 are found, with an absence of vimentin. Moreover, in the case of RCC metastases, we find high vimentin levels, whereas CK7 testing yields negative results (Fig. 3B) (3).

In all gallbladder findings with suspected malignancy, or benign lesions larger than $1 \mathrm{~cm}$, a cholecystectomy should be

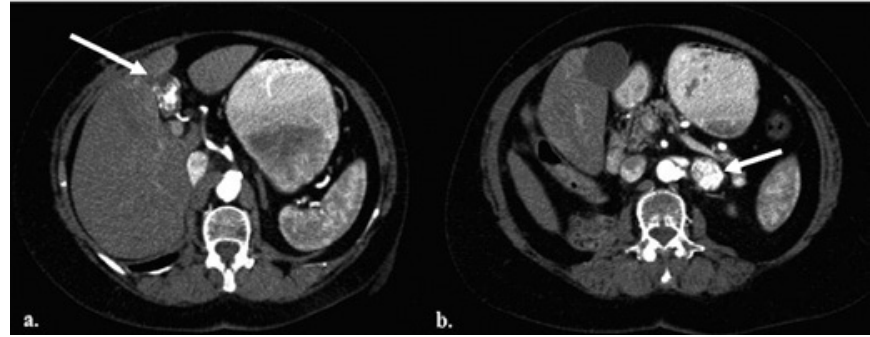

Figure 2. (A) Computed tomography (CT) image of a heterogeneous $2-3 \mathrm{~cm}$ nodule in the gallbladder with intense uptake in the arterial phase. (B) CT image of a hypervascular nodule in the left adrenal gland.
$\mathbf{A}$

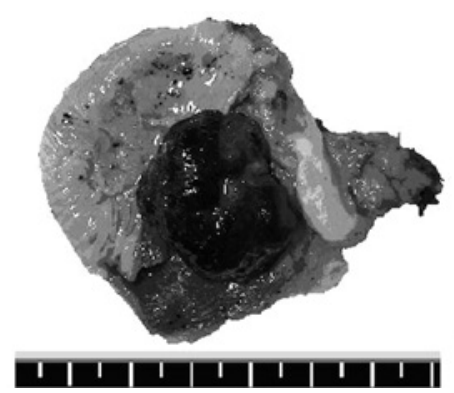

B

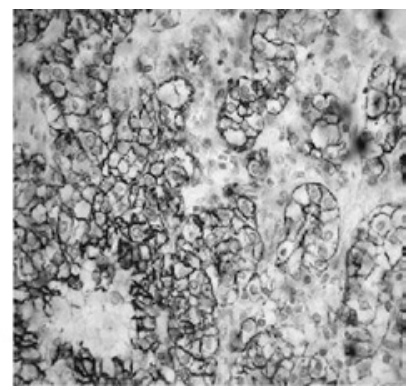

Figure 3. (A) Exophytic and polypoid lesion of $2.3 \times 1.8 \mathrm{~cm}$ in the gallbladder. (B) Immunohistochemical technique of vimentin staining. The vimentin usually stains in the mesenchyme, but in clear cell renal carcinoma this staining occurs in the cytoplasm of tumor cells.

performed to obtain a definitive diagnosis (5). Cholecystectomy with $\mathrm{R} 0$ resection has been demonstrated to be the only factor that increases survival, mainly in isolated cases of metastasis $(4,10)$. Acute cholecystitis as a clinical presentation is associated with poor prognosis (10).

The five-year survival rate following cholecystectomy for RCC is $35-50 \%$. According to Chung et al (5), 63\% of patients with one gallbladder metastasis have a two-year survival, while in the case of multiple metastases this rate decreases to $23 \%$ following cholecystectomy. In conclusion, gallbladder metastasis of RCC tumors is a rare entity, but should be borne in mind in the case of diagnosis of a gallbladder lesion during the diagnosis and/or follow-up of a primary tumor. Although radiological tests may raise clinical suspicion, a cholecystectomy should be carried out to determine the definitive diagnosis and improve survival.

\section{References}

1. Choi JB, Yoon BI, Kim SJ, Cho HJ, Hong SH, Choi YJ, Kim SW, Hwang TK and Lee JY: Changes in clinicopathological characteristics of renal cell carcinoma in the past 25 years: a single-center experience. Korean J Urol 52: 110-114, 2011.

2. Kim WJ, Chung JI, Hong JH, Kim CS, Jung SI and Yoon DK: Epidemiological study for urologic cancer in Korea (1998-2002) Korean J Urol 45: 1081-1088, 2004.

3. Nojima H, Cho A, Yamamoto H, Nagata M, Takiguchi N, Kainuma O, Souda H, Gunji H, Miyazaki A, Ikeda A, et al: Renal cell carcinoma with unusual metastasis to the gallbladder. J Hepatobiliary Pancreat Surg 15: 209-212, 2008. 
4. Fang X, Gupta N, Shen SS, Tamboli P, Charnsangavej C, Rashid A and Wang $\mathrm{H}$ : Intraluminal polypoid metastasis of renal cell carcinoma in gallbladder mimicking gallbladder polyp. Arch Pathol Lab Med 134: 1003-1009, 2010.

5. Chung PH, Srinivasan R, Linehan WM, Pinto PA and Bratslavsky G: Renal cell carcinoma with metastases to the gallbladder: Four cases from the National Cancer Institute and review of the literature. Urol Oncol 28, 2011 (Epub ahead of print).

6. Limani K, Matos C, Hut F, Gelin M and Closset J: Metastatic carcinoma of the gallbladder after a renal cell carcinoma. Acta Chir Belg 103: 233-234, 2003.

7. Barretta ML, Catalano O, Setola SV, Granata V, Marone U and D'Errico Gallipoli A: Gallbladder metastasis: spectrum of imaging findings. Abdom Imaging 36: 729-734.
8. Xie XH, Xu HX, Xie XY, Lu MD, Kuang M, Xu ZF, Liu GJ, Wang Z, Liang JY, Chen LD and Lin MX: Differential diagnosis between benign and malignant gallbladder diseases with real-time contrast-enhanced ultrasound. Eur Radiol 20: 239-248, 2010.

9. Kawahara T, Ohshiro H, Sekiguchi Z, Furuya M, Namura K, Itoh H, Sano F, Kawaji K, Hayashi N, Makiyama K, et al: Gallbladder metastasis from renal cell carcinoma. Case Rep Oncol 29: 30-34, 2010.

10. Yoon WJ, Yoon YB, Kim YJ, Ryu JK and Kim YT: Metastasis to the gallbladder: a single-center experience of 20 cases in South Korea: World J Gastroenterol 15: 4806-4809, 2009. 